\title{
m-CPBA/KOH: An Efficient Reagent for Nucleophilic Epoxidation of gem-Deactivated Olefines.
}

\author{
J. L. García Ruano, * Cristina Fajardo, Alberto Fraile and M. Rosario Martín* \\ Departamento de Química Orgánica, Universidad Autónoma de Madrid,
}

Cantoblanco, 28049-Madrid, Spain.

joseluis.garcia.ruano@uam.es

\section{Supporting Information}

(19 pages)

General Methods

Experimental Procedures and characterization data

of compounds $6,8,10,11,17-19$ and 28

${ }^{1}$ H NMR spectrum of compound 6

${ }^{1} \mathrm{H}$ NMR and ${ }^{13} \mathrm{C}$ NMR spectra of compound 7

${ }^{1} \mathrm{H}$ NMR and ${ }^{13} \mathrm{C}$ NMR spectra of compound $\mathbf{8}$

${ }^{1} \mathrm{H}$ NMR and ${ }^{13} \mathrm{C}$ NMR spectra of compound $\mathbf{1 6}$

${ }^{1} \mathrm{H}$ NMR and ${ }^{13} \mathrm{C}$ NMR spectra of compound $\mathbf{1 8}$

${ }^{1} \mathrm{H}$ NMR and ${ }^{13} \mathrm{C}$ NMR spectra of compound $\mathbf{1 9}$

${ }^{1} \mathrm{H}$ NMR and ${ }^{13} \mathrm{C}$ NMR spectra of compound $\mathbf{2 4}$

${ }^{1} \mathrm{H}$ NMR and ${ }^{13} \mathrm{C}$ NMR spectra of compound 25

${ }^{1} \mathrm{H}$ NMR and ${ }^{13} \mathrm{C}$ NMR spectra of compound 26a

${ }^{1} \mathrm{H}$ NMR and ${ }^{13} \mathrm{C}$ NMR spectra of compound $\mathbf{2 8}$

${ }^{1} \mathrm{H}$ NMR and ${ }^{13} \mathrm{C}$ NMR spectra of compound $\mathbf{3 0}$ 


\section{General Methods.}

Dichloromethane was distilled over $\mathrm{P}_{2} \mathrm{O}_{5}$. Silica gel 60 (230-400 mesh ASTM) and DC-Alufolien $60 \mathrm{~F}_{254}$ were used for flash column chromatography and analytical TLC, respectively. Melting points were determined in open capillary tubes and are uncorrected. NMR spectra were determined in $\mathrm{CDCl}_{3}$ solutions, unless otherwise is indicated, at 300 and $75 \mathrm{MHz}$ for ${ }^{1} \mathrm{H}$ and ${ }^{13} \mathrm{C} \mathrm{NMR}$ respectively; chemical shifts $(\delta)$ are reported in ppm and $J$ values are given in hertz. The IR spectra frequencies are given in $\mathrm{cm}^{-1} . m$-CPBA (73\% in water) was commercially available from AldrichSigma. Prior to its use, the water was removed dissolving the commercial product in dichloromethane and drying the organic solution over anhydrous $\mathrm{Na}_{2} \mathrm{SO}_{4}$, which was filtered off, and then the solution was evaporated to dryness under vacuum. It was titulated following the procedure reported in literature $^{1}$ to afford a purity of $84 \%$. Alkenes $\mathbf{1 2}$ and $\mathbf{1 3}$ were synthesized following the procedure reported in literature. ${ }^{2}$ Racemic sulfoxide $\mathbf{2 2}^{3}$ was synthesized following the procedure reported for the synthesis of 2-phenylsulfinylcyclopenten-1-one. ${ }^{4}$ Racemic sulfoxide $\mathbf{2 4} \mathbf{4}^{3,5}$ was synthesized from sulfoxide 22 by 1,3-dipolar cycloaddition with diazomethane and subsequent extrusion of nitrogen by heating the obtained pyrazoline. ${ }^{6}$ Although compounds $6,11,17,19$ and 28 have been previously cited in the literature, their spectroscopic data have not been reported.

2-[(4-Methylphenyl)sulfonyl]cyclopent-2-en-1-one (6): ${ }^{7}$ It was obtained following GP1 from 2[(4-methylphenyl)sulfinyl]cyclopent-2-en-1-one (22) (396 mg, $1.8 \mathrm{mmol})$ and m-CPBA (555 mg, $2.7 \mathrm{mmol}$ ) after 1 hour of reaction. Flash column chromatography (ethyl acetate-hexane, 1:2) gave pure sulfone 6 (370 mg, yield $87 \%$ ) as a white solid: mp (hexane) $126-128{ }^{\circ} \mathrm{C} .{ }^{1} \mathrm{H}$ NMR $\delta 8.46(\mathrm{t}$, $J=2.7,1 \mathrm{H}), 7.94$ and 7.33 (AA'BB' system, 4H), 2.83-2.77 (m, 2H), 2.58-2.51 (m, 2H), $2.43(\mathrm{~s}, 3 \mathrm{H})$.

Synthesis of 3-[(4-methylphenyl)sulfonyl]-5-ethoxyfuran-2(5H)-one (8): Following GP1, $t$ butyl (2E)-4,4-diethoxy-2-[(4-methylphenyl)sulfinyl]-2-butenoate ${ }^{8}(250 \mathrm{mg}, 0.68 \mathrm{mmol})$ was oxidized with $\mathrm{m}$-CPBA (279 $\mathrm{mg}, 1.36 \mathrm{mmol})$ after 2 hours of reaction. The obtained residue was a 
colorless oil corresponding to pure $t$-butyl (2E)-4,4-diethoxy-2-[(4-methylphenyl)sulfonyl]-2butenoate. ${ }^{9}$ This compound was transformed into furanone $\mathbf{8}$ following the procedure reported by us for the synthesis of $\left(5 R, S_{\mathrm{S}}\right)-3$-[(4-methylphenyl)sulfinyl]-5-ethoxyfuran-2(5H)-one. ${ }^{10}$ The residue as a colorless oil corresponding to pure furanone 8, yield 96\%. Further purification was unsuccessful. IR (film): 1781. ${ }^{1} \mathrm{H}$ NMR $\delta 7.94$ and 7.36 (AA'BB' system, 4H), 7.84 (d, $\left.J=0.8,1 \mathrm{H}\right), 5.93$ (d, $J=0.8$, 1H), $3.90(\mathrm{~m}, 1 \mathrm{H}), 3.76(\mathrm{~m}, 1 \mathrm{H}), 2.43(\mathrm{~s}, 3 \mathrm{H}), 1.24(\mathrm{t}, J=6.9,3 \mathrm{H}) .{ }^{13} \mathrm{C}$ NMR $\delta 162.7(\mathrm{C}), 153.6$ $(\mathrm{CH}), 146.2,139.7$ and $134.3(\mathrm{C}), 130.0,129.2$ and $100.4(\mathrm{CH}), 67.2\left(\mathrm{CH}_{2}\right), 21.7$ and $14.8\left(\mathrm{CH}_{3}\right)$.

Methyl (2E)-2-[(4-methylphenyl)sulfonyl]-3-phenylacrylate (11): ${ }^{11}$ It was obtained following GP1 from methyl (2E)-2-[(4-methylphenyl)sulfinyl]-3-phenylacrylate ${ }^{12}(67 \mathrm{mg}, 0.22 \mathrm{mmol})$ and $\mathrm{m}$ CPBA (69 mg, $0.33 \mathrm{mmol}$ ) after 2.5 hours of reaction. Flash column chromatography (ethyl acetatehexane, 1:5) gave pure sulfone 11 (58 mg, yield 84\%) as a white solid (ethyl ether:hexane) mp 84-86 ${ }^{\circ} \mathrm{C} .{ }^{1} \mathrm{H}$ NMR $\delta 7.95(\mathrm{~s}, 1 \mathrm{H}), 7.40(\mathrm{~m}, 5 \mathrm{H}), 7.83$ and 7.35 (AA'BB' system, 4H), $3.75(\mathrm{~s}, 3 \mathrm{H}), 2.44$ (s, $3 \mathrm{H}) .{ }^{13} \mathrm{C}$ NMR $\delta 163.8$ and $144.8(\mathrm{C}), 143.5(\mathrm{CH}), 136.9,134.9$ and $131.6(\mathrm{C}), 131.3,129.8,129.7$, 128.9 and $128.6(\mathrm{CH}), 52.9$ and $21.7\left(\mathrm{CH}_{3}\right)$. Anal. Calcd for $\mathrm{C}_{17} \mathrm{H}_{16} \mathrm{O}_{4} \mathrm{~S}: \mathrm{C}$ 64.54, H 5.10, S 10.14. Found: C 64.40, H 5.14, S 9.92.

Ethyl 2-oxo-6-oxabicyclo[3.1.0]hexane-1-carboxylate (17): ${ }^{13}$ It was obtained following GP3 from ethyl 5-oxocyclopent-1-en-1-carboxylate $(\mathbf{1 2})^{2}(53 \mathrm{mg}, 0.34 \mathrm{mmol}), \mathrm{m}$-CPBA (141 mg, 0.68 mmol) and $\mathrm{KOH}(68 \mathrm{mg}, 1.02 \mathrm{mmol})$ after 50 minutes of reaction. Flash column chromatography (ethyl acetate-hexane, 1:4) gave pure epoxide $17\left(39 \mathrm{mg}\right.$, yield 66\%) as a colorless oil. ${ }^{1} \mathrm{H}$ NMR $\delta$ $4.29(\mathrm{q}, J=7.1,2 \mathrm{H}), 4.14(\mathrm{~d}, J=1.2,1 \mathrm{H}), 2.48-2.07(\mathrm{~m}, 4 \mathrm{H}), 1.31(\mathrm{t}, J=7.1,3 \mathrm{H}) .{ }^{13} \mathrm{C}$ NMR $\delta 202.9$ and $163.9(\mathrm{C}), 64.7(\mathrm{CH}), 62.1\left(\mathrm{CH}_{2}\right), 59.1(\mathrm{C}), 32.1$ and $22.2\left(\mathrm{CH}_{2}\right), 14.0\left(\mathrm{CH}_{3}\right)$. Anal. Calcd. for $\mathrm{C}_{8} \mathrm{H}_{10} \mathrm{O}_{4}$ : C 56.47, H 5.92. Found: C 56.27, H 6.01.

Ethyl 2-oxo-7-oxabicyclo[4.1.0]heptane-1-carboxylate (18): ${ }^{14}$ It was obtained following GP3 from ethyl 6-oxocyclohex-1-en-1-carboxylate $(\mathbf{1 3})^{2}(69 \mathrm{mg}, 0.41 \mathrm{mmol}), \mathrm{m}$-CPBA (169 mg, 0.82 $\mathrm{mmol})$ and $\mathrm{KOH}(81 \mathrm{mg}, 1.23 \mathrm{mmol})$ after 50 minutes of reaction. Flash column chromatography (ethyl acetate-hexane, 1:4) gave pure epoxide $18\left(67 \mathrm{mg}\right.$, yield 89\%) as a colorless oil. ${ }^{1} \mathrm{H}$ NMR $\delta$ $4.27(\mathrm{q}, J=7.1,1 \mathrm{H}), 4.26(\mathrm{q}, J=7.1,1 \mathrm{H}), 3.66(\mathrm{~m}, 1 \mathrm{H}), 2.55(\mathrm{dt}, J=17.0$ and $4.2,1 \mathrm{H}), 2.31(\mathrm{~m}, 1 \mathrm{H})$, 
$2.15(\mathrm{ddd}, J=17.0,11.3$ and 5.9, $1 \mathrm{H}), 2.09$ (dddd, $J=15.2,10.7,5.7$ and 1.4, $1 \mathrm{H}), 1.90-1.67(\mathrm{~m}, 2 \mathrm{H})$, $1.29(\mathrm{t}, J=7.1,3 \mathrm{H}) .{ }^{13} \mathrm{C}$ NMR $\delta 199.4$ and $166.0(\mathrm{C}), 62.0\left(\mathrm{CH}_{2}\right), 59.9(\mathrm{CH}), 58.7(\mathrm{C}), 37.3,22.8$ and $16.5\left(\mathrm{CH}_{2}\right), 14.0\left(\mathrm{CH}_{3}\right)$.

Diethyl 3-methyloxirane-2,2-dicarboxylate (19). ${ }^{15}$ It was obtained following GP3 from diethyl ethylidenemalonate (14) (101 mg, $0.54 \mathrm{mmol}), m$-CPBA (446 mg, $2.17 \mathrm{mmol})$ and KOH (239 mg, $3.6 \mathrm{mmol}$ ) after 2 hours of reaction. Flash column chromatography (ethyl acetate-hexane, 1:10) gave pure epoxide 19 (93 mg, yield 85\%) as a colorless oil. IR (film): 1750, 1372, 1239, 1081, $1025 .{ }^{1} \mathrm{H}$ NMR $\delta 4.34$ (2q, $J=7.1,2 \mathrm{H}), 4.26(\mathrm{q}, J=7.1,2 \mathrm{H}), 3.60$ (q, $J=5.5,1 \mathrm{H}), 1.37(\mathrm{~d}, J=5.5,3 \mathrm{H}), 1.33$ (t, $J=7.1,3 \mathrm{H}), 1.30(\mathrm{t}, J=7.1,3 \mathrm{H}) .{ }^{13} \mathrm{C} \mathrm{NMR} \delta 165.9$ and $164.7(\mathrm{C}), 62.4$ and $\left(\mathrm{CH}_{2}\right), 60.4(\mathrm{C}), 58.3$ (CH), 14.0, 13.9 and $13.7\left(\mathrm{CH}_{3}\right)$.

Diethyl (cyclohex-3-en-1-ylmethylene)malonate (28): ${ }^{16}$ It was synthesized by Knovenagel reaction from 1,2,3,6,-tetrahydrobenzaldehyde and diethyl malonate following the procedure described in reference 17. Purification by flash column chromatography (ethyl ether-hexane, 1:10) gave diene 28 as a colorless oil. Yield 82\%. ${ }^{1} \mathrm{H}$ NMR $\delta 6.87(\mathrm{~d}, J=10.5,1 \mathrm{H})$, 5.76-5.60 (m, 2H), $4.29(2 \mathrm{q}, J=7.1,2 \mathrm{H}), 4.23(\mathrm{q}, J=7.1,2 \mathrm{H}), 2.67(\mathrm{~m}, 1 \mathrm{H}), 2.21-2.02(\mathrm{~m}, 3 \mathrm{H}), 1.98-1.73(\mathrm{~m}, 2 \mathrm{H}), 1.50$ $(\mathrm{m}, 1 \mathrm{H}), 1.31(\mathrm{t}, J=7.1,3 \mathrm{H}), 1.29(\mathrm{t}, J=7.1,3 \mathrm{H}) .{ }^{13} \mathrm{C} \mathrm{NMR} \delta 165.4$ and $163.9(\mathrm{C}), 152.4(\mathrm{CH}), 127.4$ (C), 126.7 and $124.6(\mathrm{CH}), 61.1$ and $61.0\left(\mathrm{CH}_{2}\right), 34.6(\mathrm{CH}), 29.9,27.4$ and $23.6\left(\mathrm{CH}_{2}\right), 13.9\left(\mathrm{CH}_{3}\right)$.

2-Menthyloxy-1-phenylsulfonyl-3,6-dioxabicyclo[3.1.0]hexan-4-ones (21a and 21b): They were synthesized by GP2 modified as follows: sulfone $\mathbf{2 0}^{18}(22 \mathrm{mg}, 0.058 \mathrm{mmol})$ was oxidated using $m$-CPBA (24 mg, $0116 \mathrm{mmol})$ and $\mathrm{K}_{2} \mathrm{CO}_{3}(8 \mathrm{mg}, 0.058 \mathrm{mmol})$. The reaction time was 8 hours. ${ }^{1} \mathrm{H}$ NMR analysis of the crude mixture showed a mixture of the starting material and epoxides $\mathbf{2 1}$ in a 51: 49 ratio. All attempts to separate theses compounds were unsuccessful. The representative signals of epoxides 21 were identified in the spectra of the crude mixture. ${ }^{1} \mathrm{H}$ NMR $(200 \mathrm{MHz}) \delta$ $6.10(\mathrm{~s}, 1 \mathrm{H}), 5.75(\mathrm{~s}, 1 \mathrm{H}), 4.22(\mathrm{~s}, 1 \mathrm{H}), 4.02(\mathrm{~s}, 1 \mathrm{H})$. 


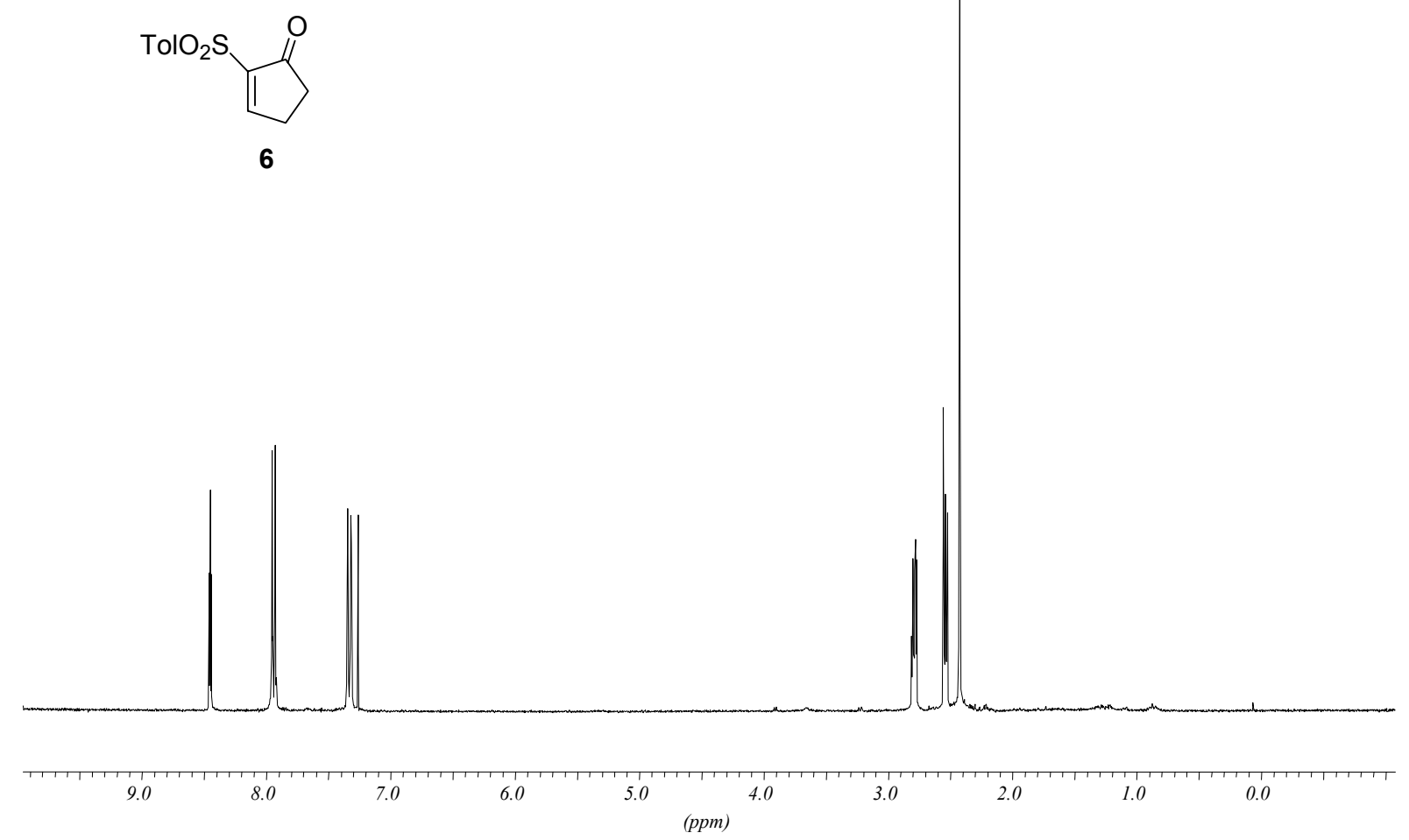



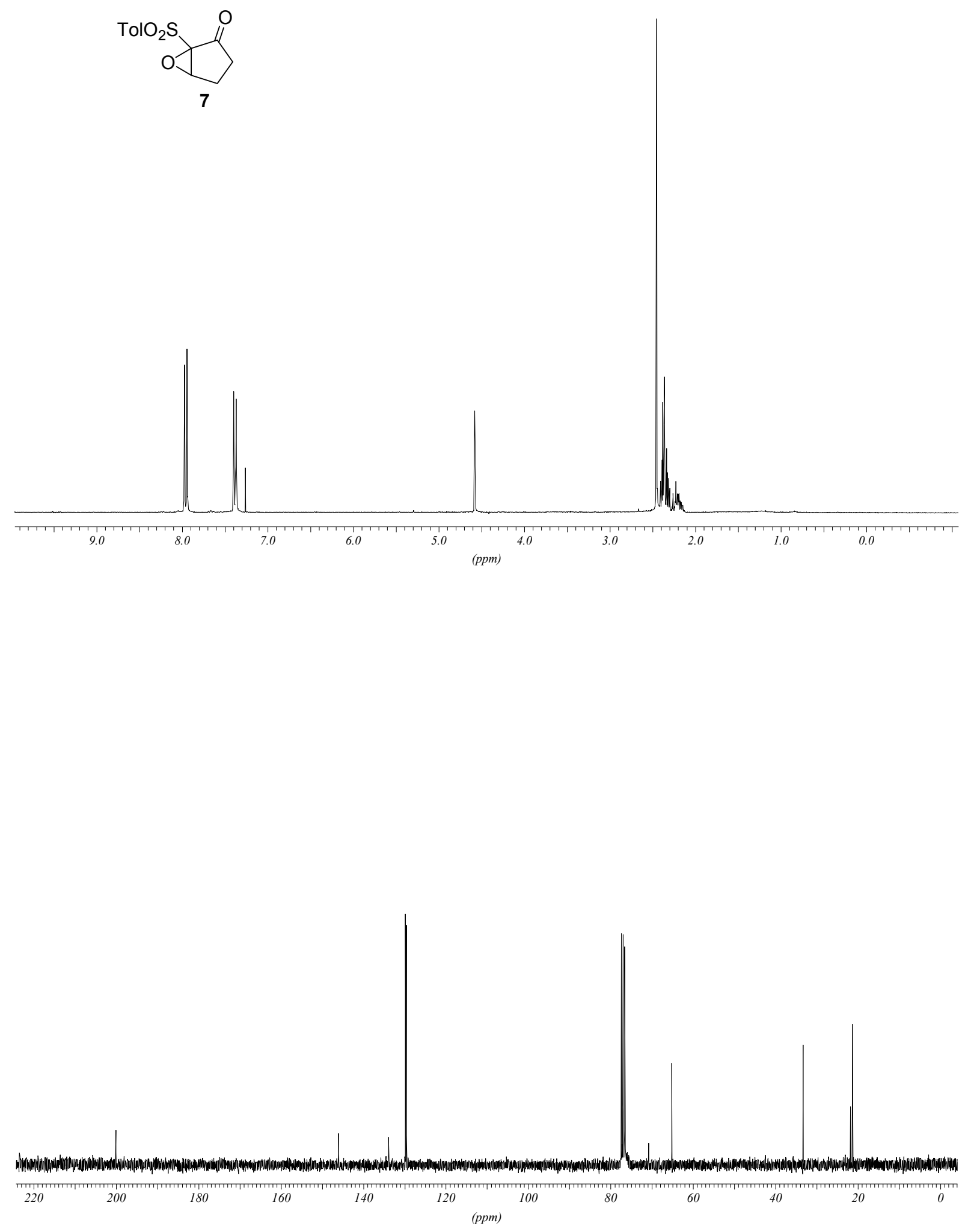

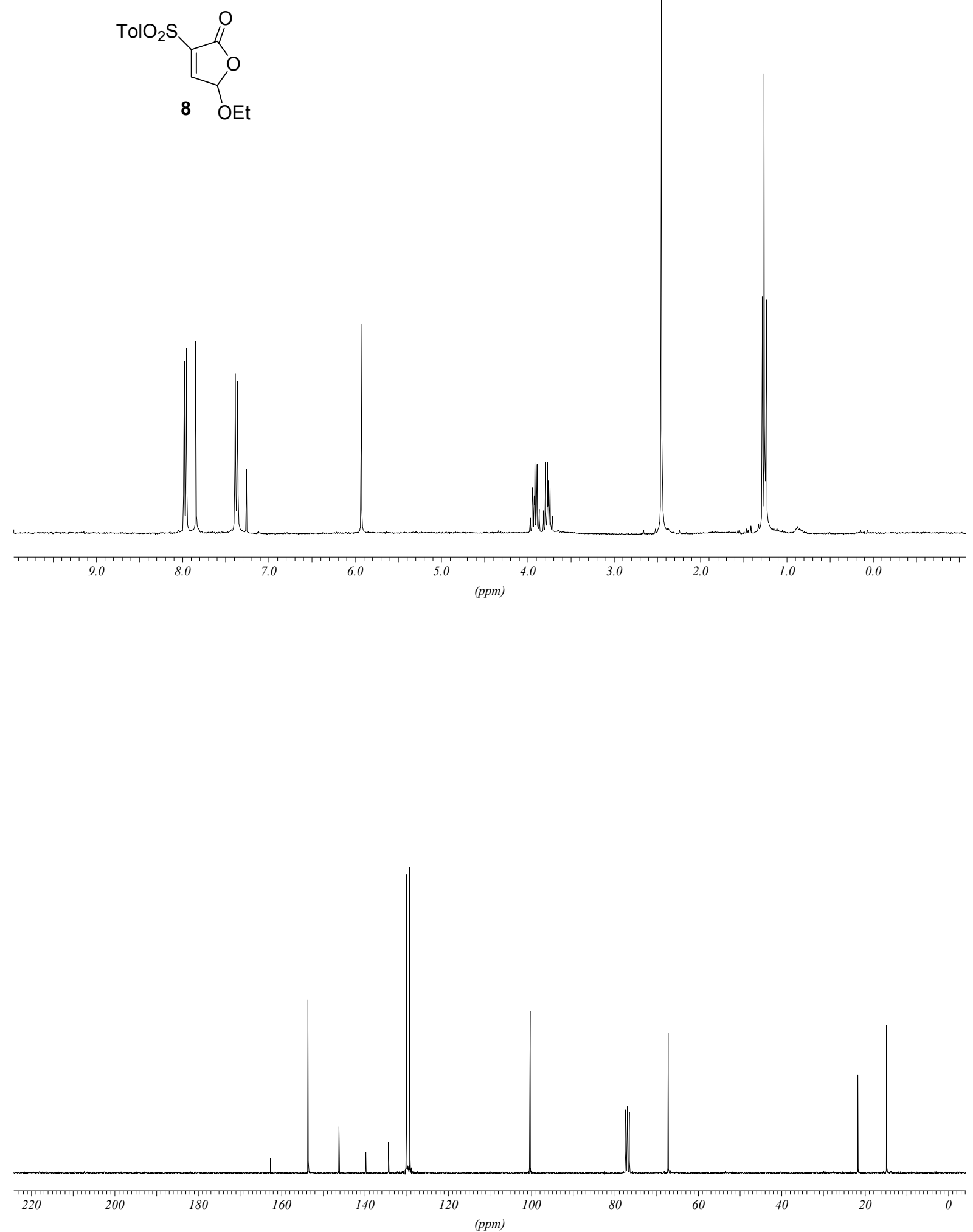

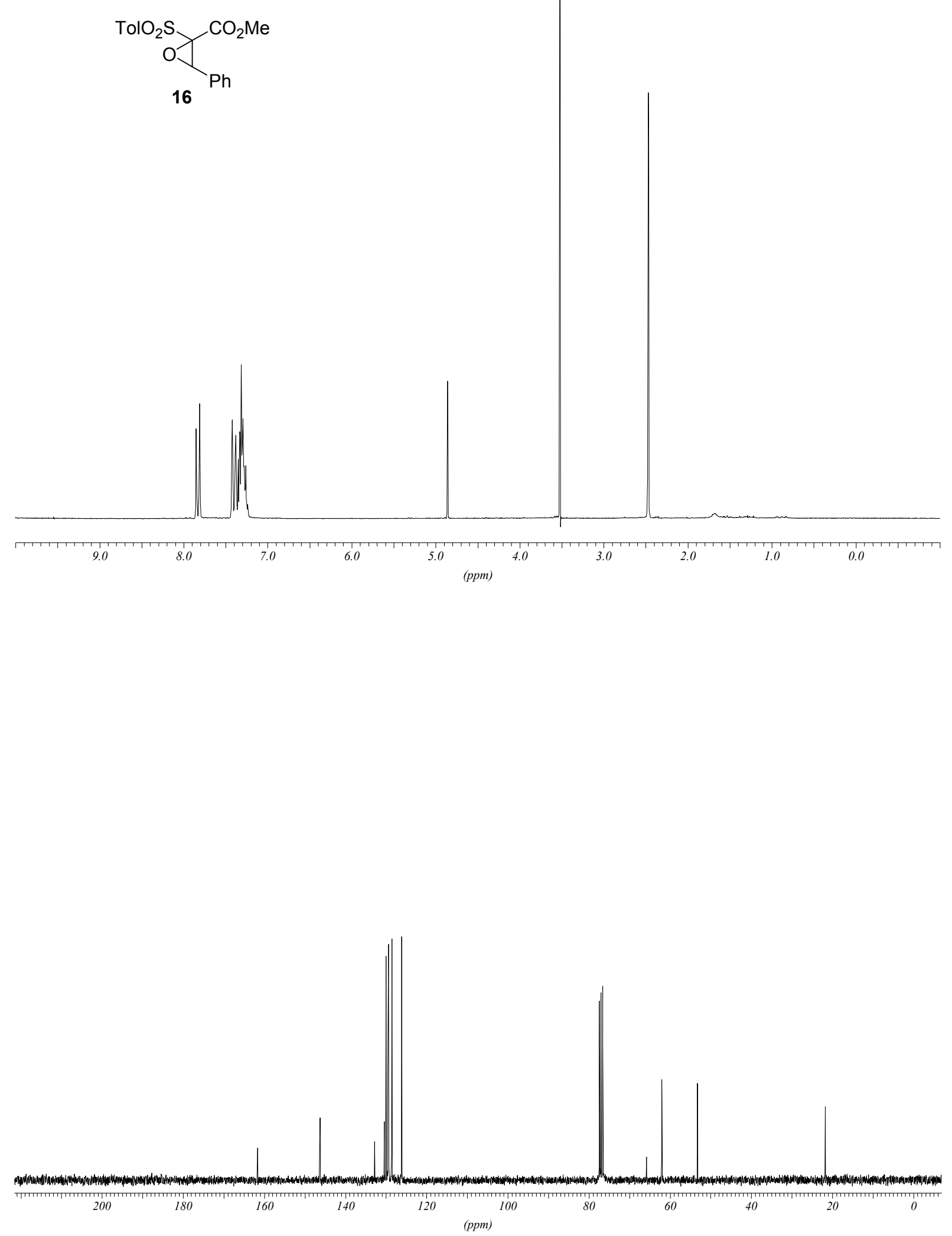


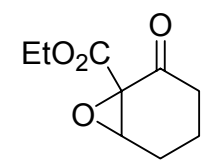

18
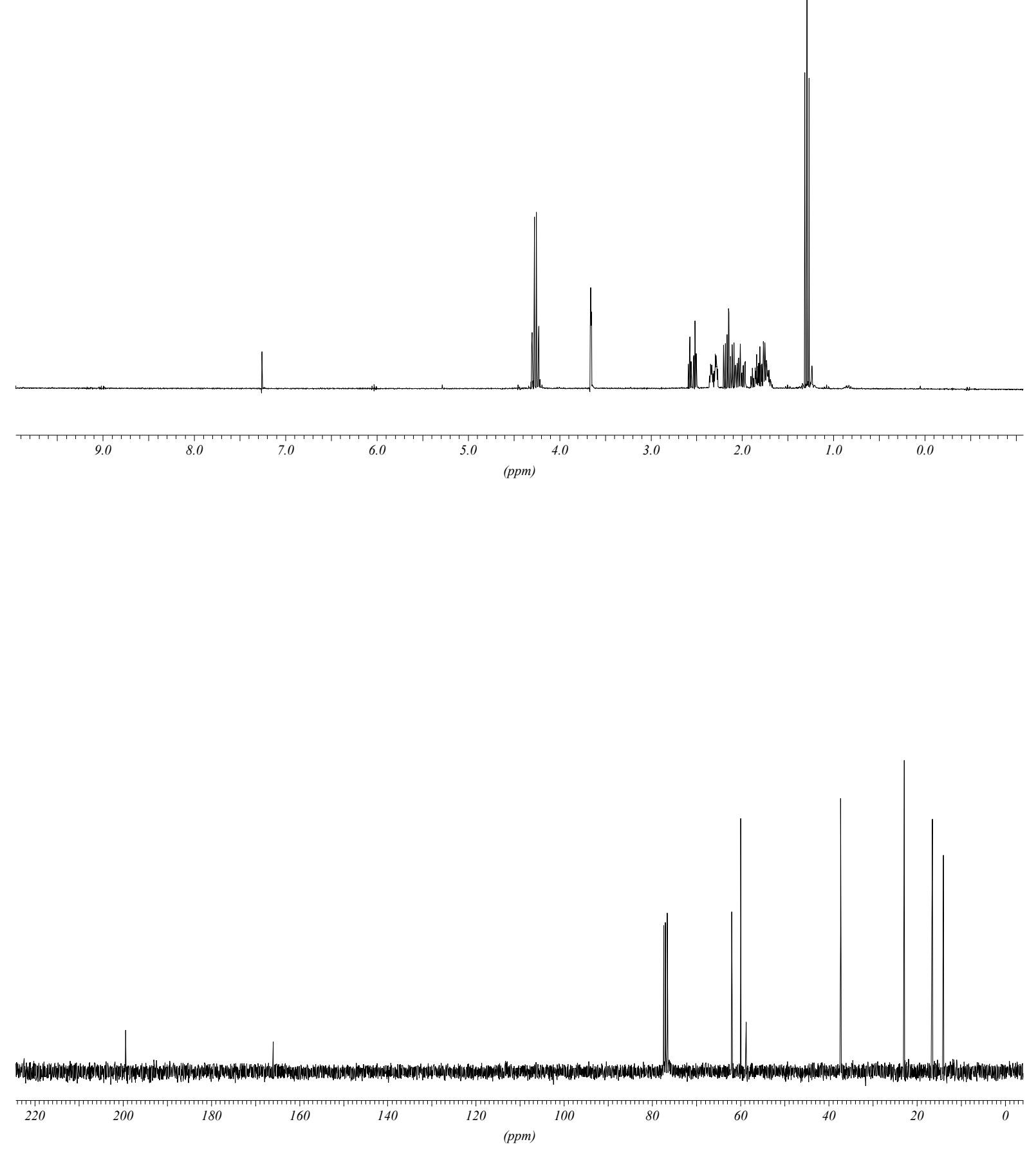
${ }_{\mathrm{Me}}^{\mathrm{EtO}_{2} \mathrm{C}} \mathrm{CO}_{2}^{\mathrm{Et}}$

19
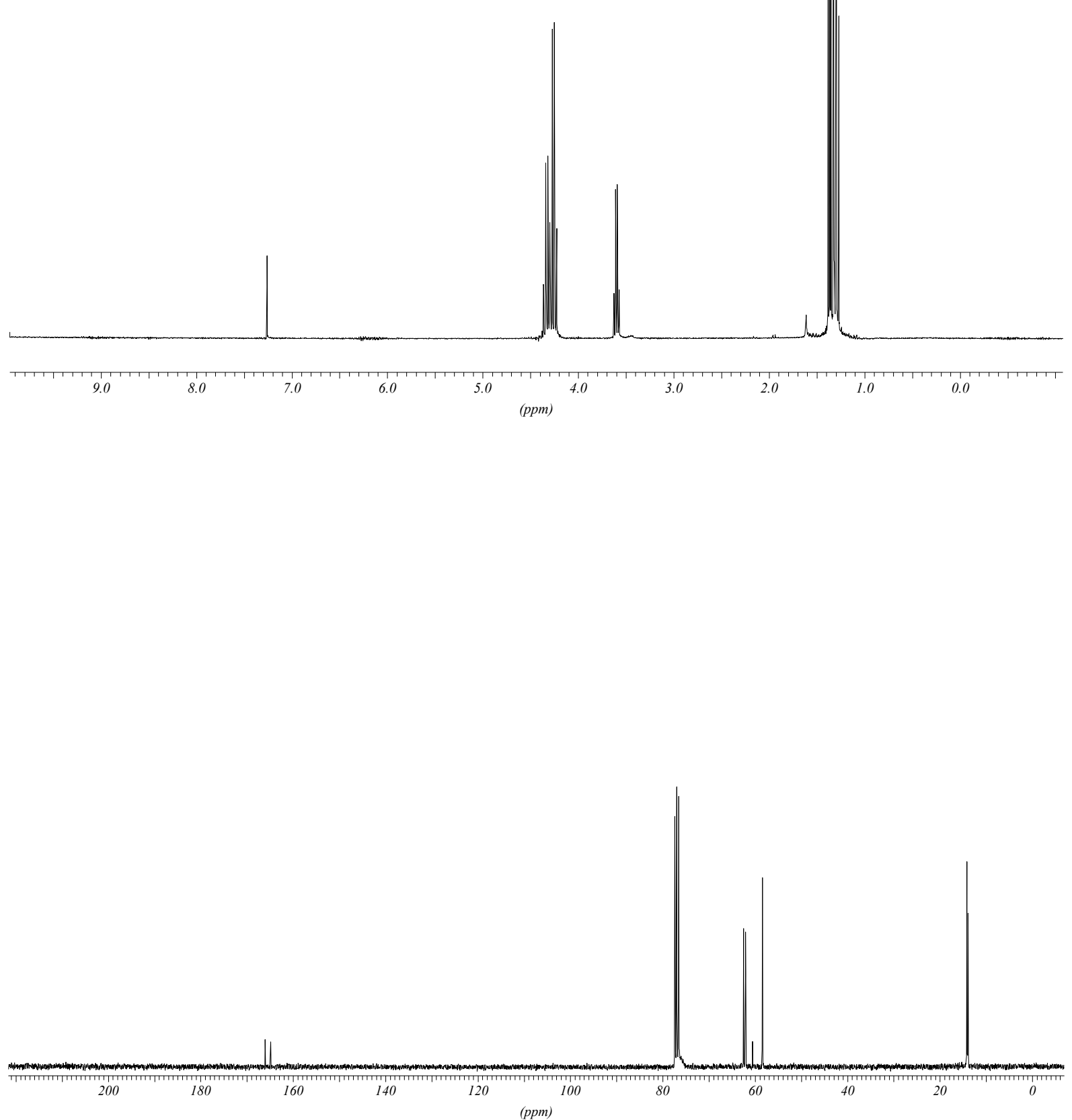

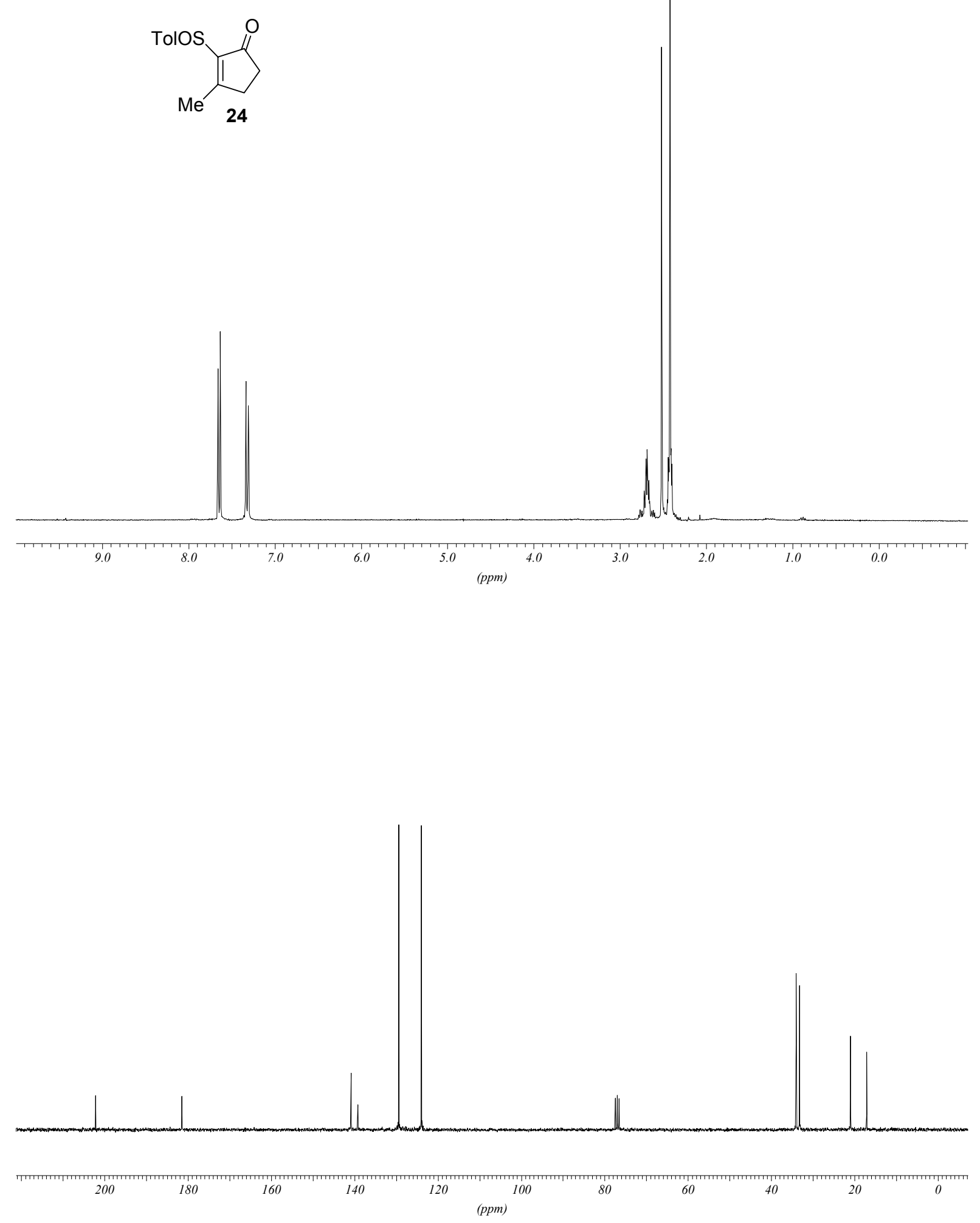

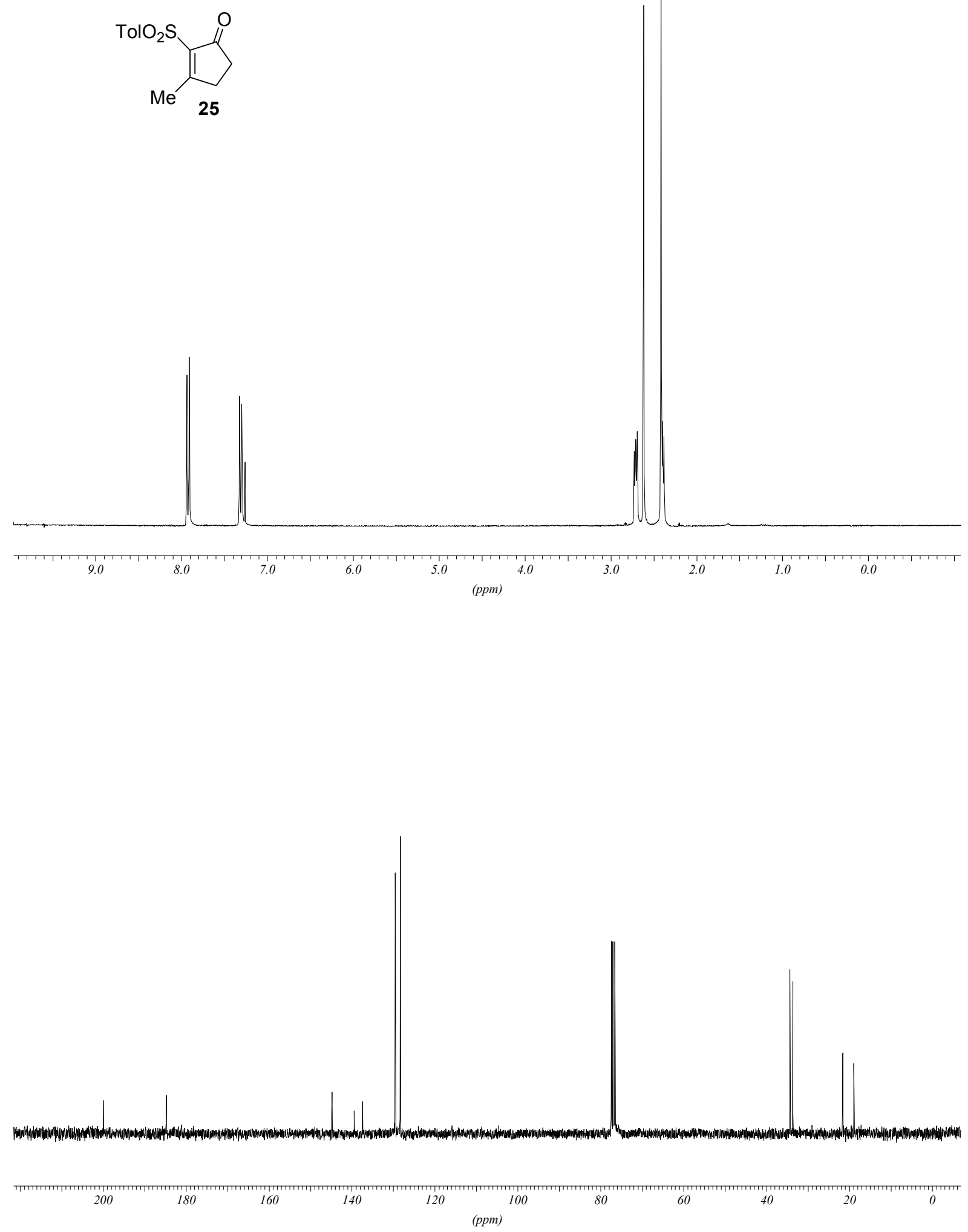

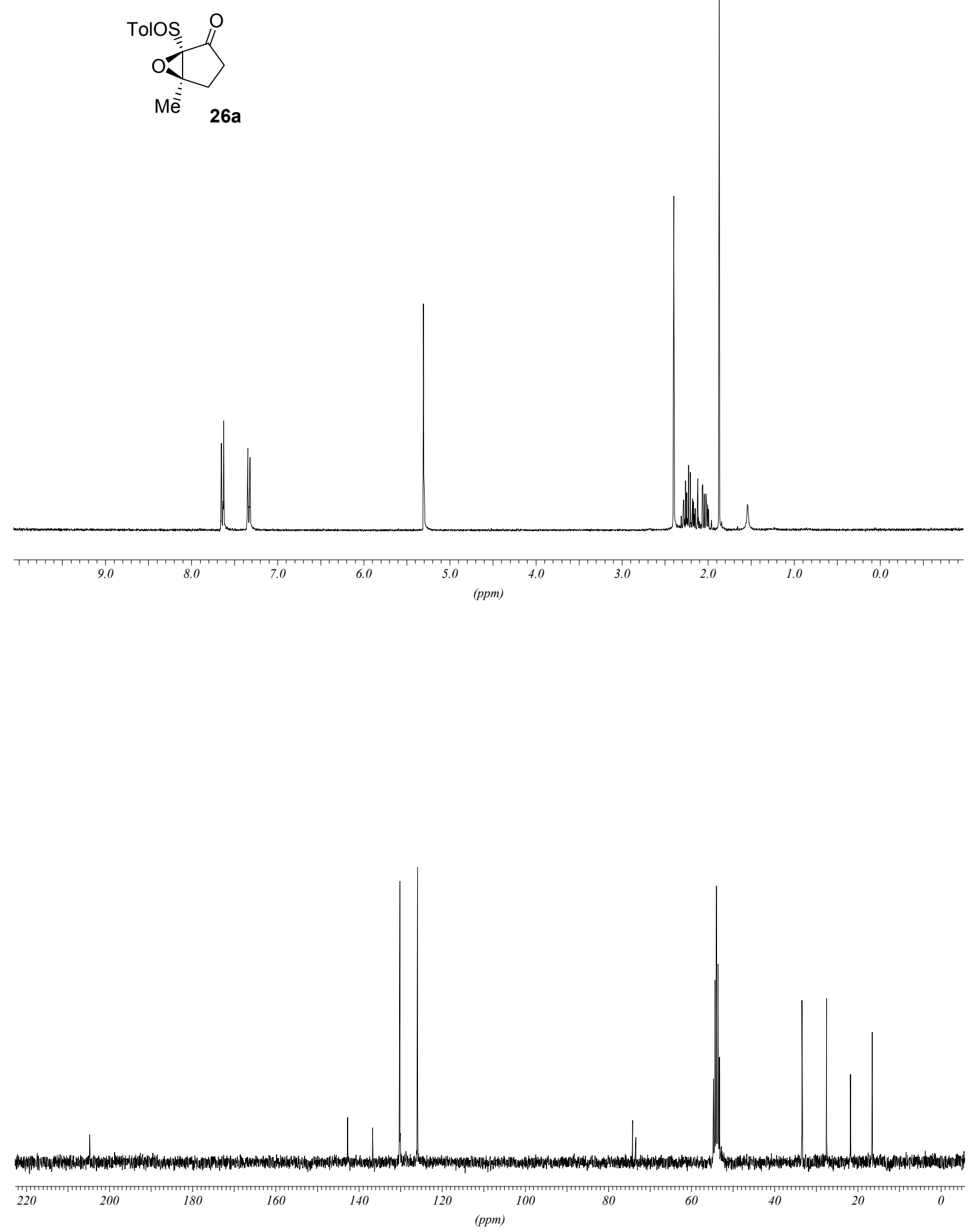
<smiles>CCOC(=O)C(=CC1CC=CCC1)C(=O)OCC</smiles>

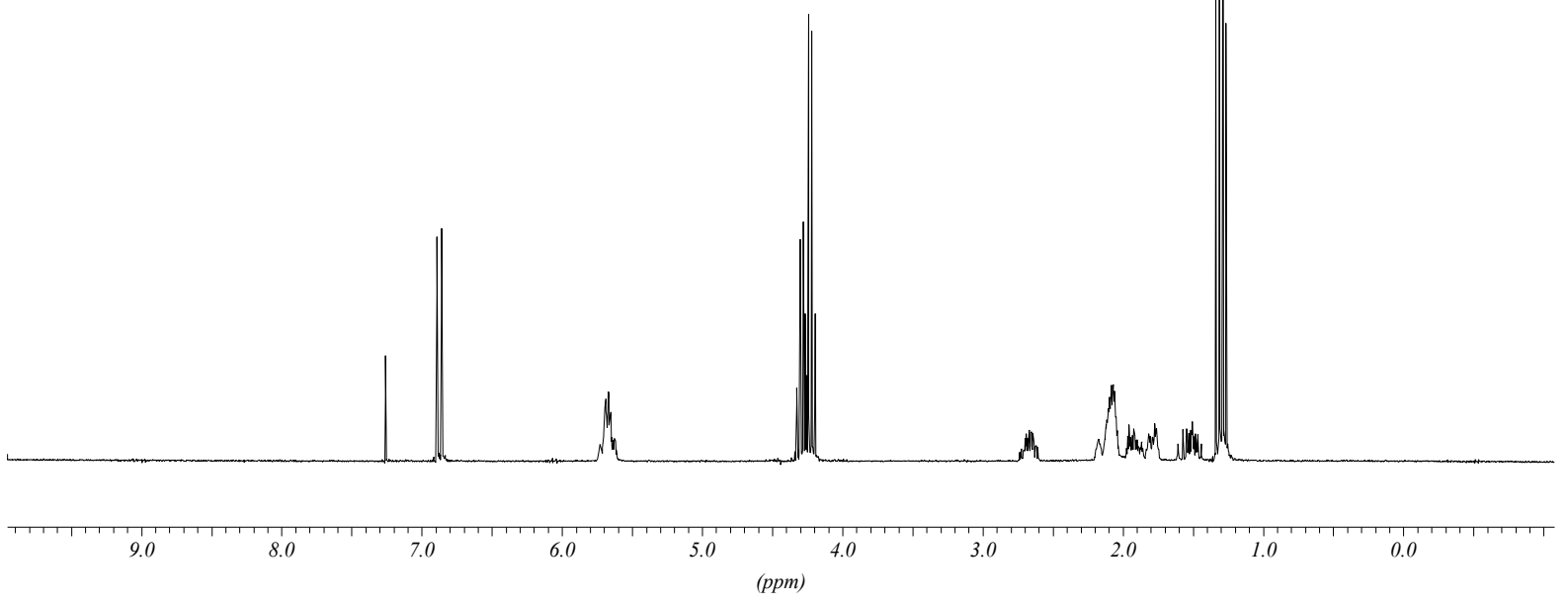

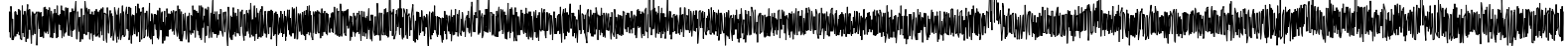

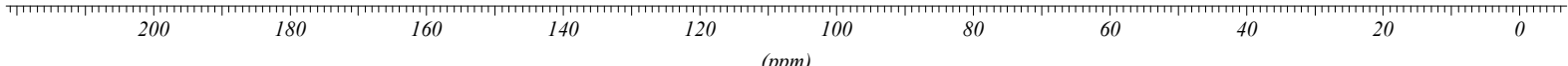


<smiles>CCOC(=O)C1(C(=O)OCC)OC1C1CC=CCC1</smiles>

30
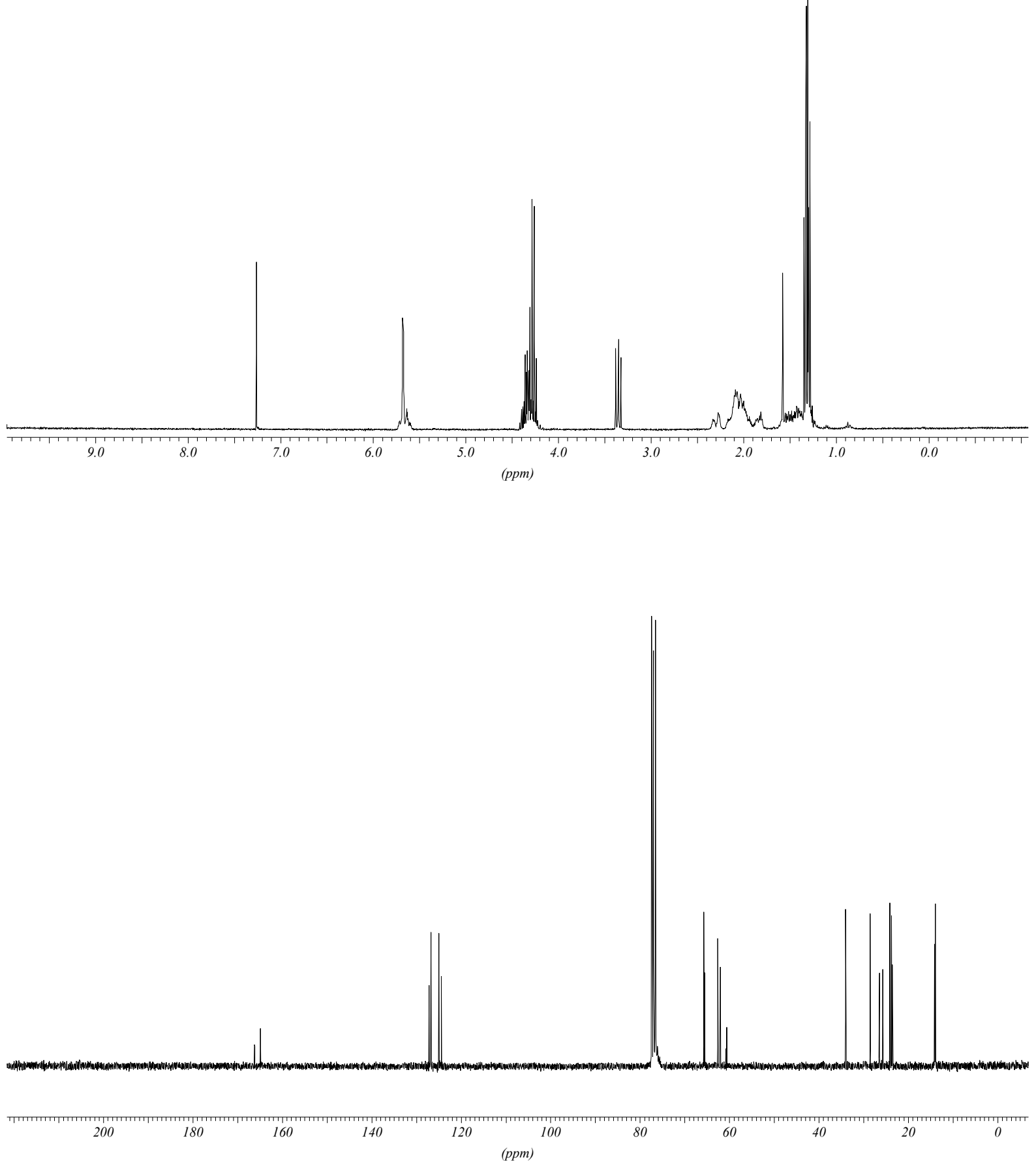

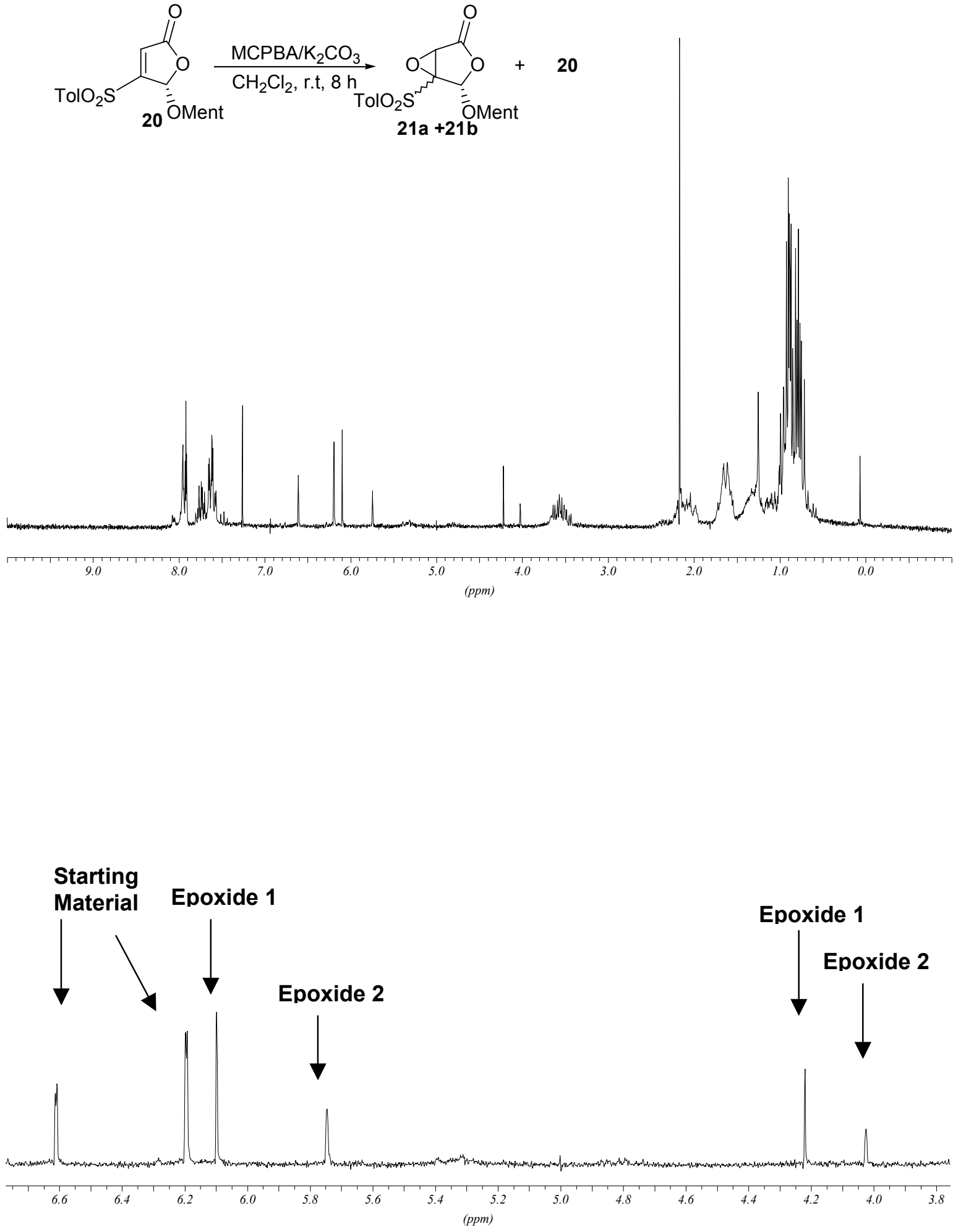
Figure 1: X-ray structure of compound $( \pm)-23 a$.

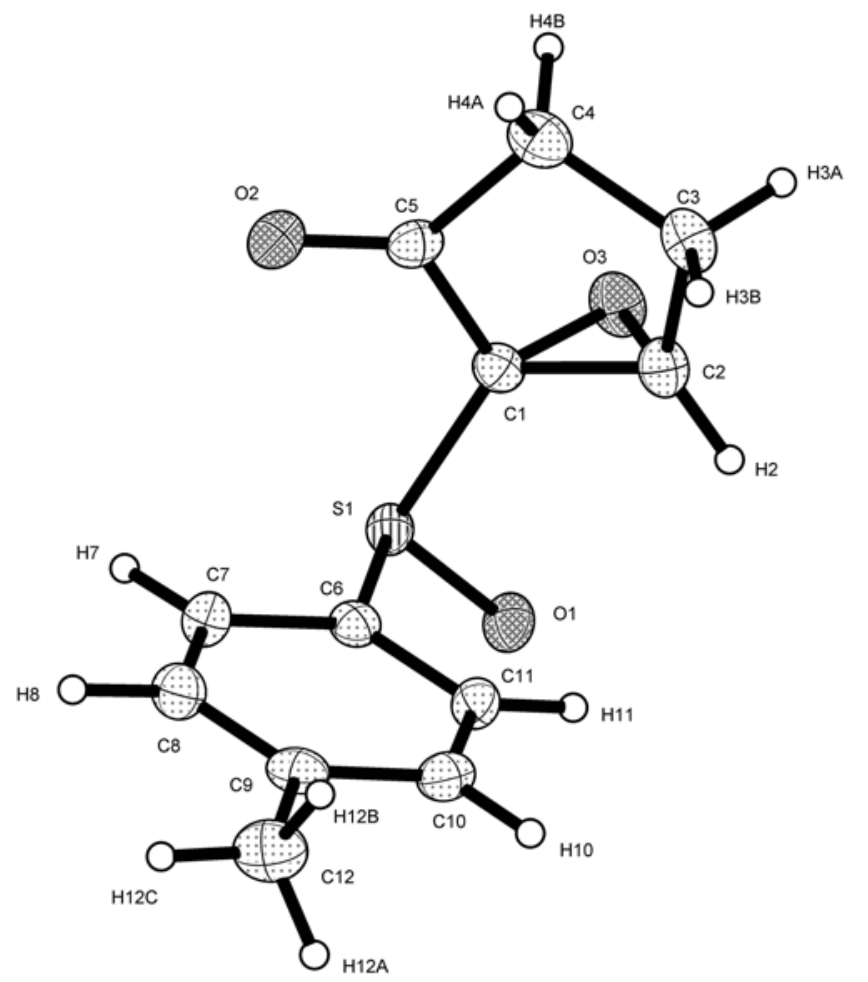

Figure 2: X-ray structure of compound ( $( \pm)-26 b$.

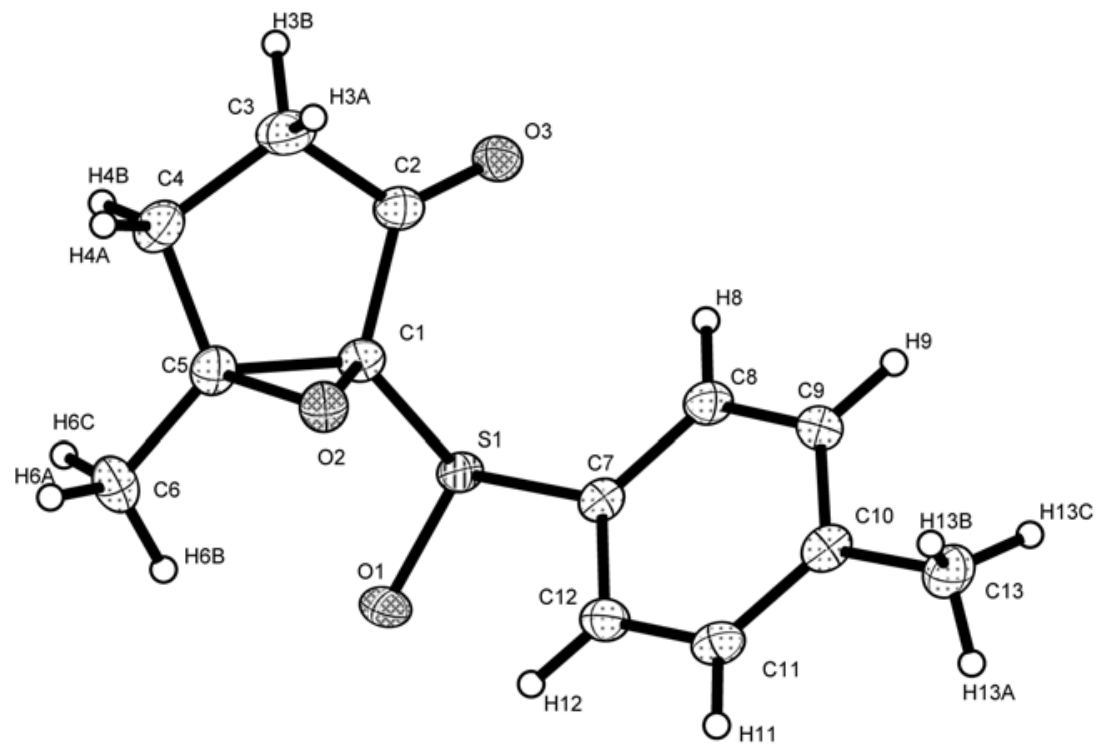


1 McDonald, R. N.; Steppel, R. N.; Dorsey, J. E. Org. Synth. 1970, 50, 15-18.

2 a) Bernardi, A.; Karamfilova, K. Sanguinetti, S.; Scolastico, C. Tetrahedron 1997, 53, 1300913026. b) Liotta, D.; Barnum, C.; Puleo, R.; Zima, G.; Bayer, C.; Kezar, H. S. J. Org. Chem. 1981, $46,2920-2923$.

3 Hulce, M.; Mallamo, J. P.; Frye, L. L.; Bogan, T. P.; Posner, G. H. Org. Synth. 1985, 64, 196206.

4 Yechezkel, T.; Ghera, E.; Ostercamp, D.; Hassner, A. J. Org. Chem. 1995, 60, 5135 and references cited therein.

5 Posner, G. H.; Kogan, T. P.; Hulce, M. Tetrahedron Lett. 1984, 25, 383-386.

6 Unpublished results.

7 Posner, G. H.; Switzer, C. J. Am. Chem. Soc. 1986, 108, 1239-1244.

8 García, J. L.; Fraile, A.; Martín, M. R. Tetrahedron 1999, 55, 14491-14500.

$9 t$-Butyl (2E)-4,4-diethoxy-2-[(4-methylphenyl)sulfonyl]-2-butenoate: Yield 99\% IR (film): 1726, 1598, 1456, 1234, 1154, 1090. ${ }^{1} \mathrm{H}$ NMR $\delta 7.71$ and 7.26 (AA'BB' system, 4H), $7.04(\mathrm{~d}, J=5.9,1 \mathrm{H})$, $5.41(\mathrm{~d}, J=5.9,1 \mathrm{H}), 3.65-3.40(\mathrm{~m}, 4 \mathrm{H}), 2.35(\mathrm{~s}, 3 \mathrm{H}), 1.32(\mathrm{~s}, 9 \mathrm{H}), 1.12(\mathrm{t}, J=7.0,6 \mathrm{H}) .{ }^{13} \mathrm{C} \mathrm{NMR} \delta$ 160.2 and $144.6(\mathrm{C}), 144.5(\mathrm{CH}), 139.8$ and $136.6(\mathrm{C}), 129.5,128.5$ and $100.0(\mathrm{CH}), 84.1(\mathrm{C}), 61.9$ $\left(\mathrm{CH}_{2}\right), 27.7,21.5$ and $15.1\left(\mathrm{CH}_{3}\right)$.

10 Carretero, J. C.; García Ruano, J. L.; Lorente, A.; Yuste, F. Tetrahedron: Asymmetry 1993, 4, 177.

11 Reedy, G. R.; Gupta, S. V. S. A. K.; Reedy, D. B.; Seenaiah, B. J. Indian. Chem. Soc. 1992, 69, 396-397.

12 Matsuyama, H.; Itoh, N.; Kamigata, N. Chem. Lett. 1990, 9, 1547-1550. 
13 Ensley, H. E.; Balakrishnan, P.; Ugarkar, B. Tetrahedon Lett. 1983, 24, 5189-8192.

14 Christoffers, J. J. Org. Chem. 1999, 64, 7668-7669.

15 Payne, G. B. J. Org. Chem. 1959, 24, 2048-2049. R. M.; Fritz, C. D.; Manning, D. T.; Wheeler, T. N.; Cooke, Anson, R. 1992, US 5123951 A, CA: 118:59427.

16 Papadakis, P E.; Haven, G. J. Pharm. Sci. 1956, 55, 1016-1018.

17 Tietze, L. F.; Beifuss, U.; Ruther, M. J. Org. Chem. 1989, 54, 3120-3129.

18 García Ruano, J. L.; Bercial, F.; Fraile, A.; Martín Castro, A. M.; Martín, M. R. Tetrahedron: Asymmetry 2000, 11, 4737-4752. 